\title{
PARTITIONS WITH PARTS APPEARING A SPECIFIED NUMBER OF TIMES
}

\author{
ELMO MOORE
}

ABSTRACT. Restrictions of this type, which are known to produce partition identities when applied to all the parts, are shown to produce partition identities when only applied to certain parts.

1. Introduction. Let $N$ be the set of natural numbers and $A, B$ and $S$ subsets of $N$. Denote by $S(n)$ the number of partitions of $n$ into parts which are el ements of $S$, and by $A^{\prime}(n, B)$ the number of partitions of $n$ where parts which are elements of $B$ each appear exactly $m$ times for some $m \in A$, while parts which are not elements of $B$ may appear any number of times. For example, if $2,3 \in A$ and $4,5 \in B, 6 \notin B$, then $6+5+$ $5+4+4+4$ is a partition of 28 of the type enumerated by $A^{\prime}(28, B)$.

$A$ is admissible relative to $B$ if there exists an $S$ such that $A^{\prime}(n, B)=$ $S(n)$ for every $n \in N$. In this case, $S$ corresponds to $A$. For example, previous results by this author [4] state that $A$ is admissible relative to $N$ if it is one of the following, where $k, d \in N$ :

$$
\begin{aligned}
& A=\{m \mid m<(2 d-1) k \text { and } m \neq(2 i-1) k+j \text { with } \\
& \qquad 1 \leq i \leq d-1,0 \leq j \leq k-1\}, \quad \text { or } \\
& A=\{m \mid m \neq(2 i-1) k+j \text { with } 1 \leq i \leq d, 0 \leq j \leq k-1\}, \quad \text { or } \\
& A=\{m \mid m \neq(2 i-1) k+j \text { with } 1 \leq i, 0 \leq j \leq k-1\} .
\end{aligned}
$$

The purpose of this paper is to show that these sets are admissible relative to $B$ for $B \neq N$. In particular, for each set $A$, there is a class of sets such that $A$ is admissible relative to $B$ for every $B$ in this class. In each case, $N$ is a member of the class and, thus, these results generalize the earlier ones.

\section{Results.}

Theorem 1. Let $k, d \in N, B \subseteq N$ such that $B \cap 2 d B \subseteq 2 B \subseteq B \cup 2 d B$. Then the number of partitions of $n$, where no part which is an element of $B$

Received by the editors October 5, 1973.

AMS (MOS) subject classifications (1970). Primary 10A45; Secondary $10 \mathrm{~J} 20$.

Key words and phrases. Partitions, generating functions. 
appears exactly $(2 i-1) k+j$ times with $1 \leq i \leq d-1,0 \leq j \leq k-1$ or more than $(2 d-1) k-1$ times, is equal to the number of partitions of $n$ into parts which are not of the form $k t$ with $t \in[(B \cup 2 d B)-2 B] \cup[B \cap 2 d B]$.

A result by Glaisher [3] states that, for every $k \in N$, the set $A=\{i\}_{i=1}^{k-1}$ is admissible relative to $N$. Letting $d=1$ in Theorem 1 , we find that this set is admissible relative to $B$ for any $B$ whatsoever.

Corollary 1. Let $k \in N, B \subseteq N$. Then the number of partitions of $n$, where parts which are elements of $B$ appear at most $k-1$ times, is equal to the number of partitions of $n$ into parts which are not of the form $k t$ with $t \in B$.

Theorem 2. Let $k, d \in N, B \subseteq N$ such that $2 B \cap(2 d+1) B \subseteq B \cap$ $(4 d+2) B \subseteq 2 B \cup(2 d+1) B \subseteq B \cup(4 d+2) B$. Then the number of partitions of $n$, where no part which is an element of $B$ appears exactly $(2 i-1) k+j$ times with $1 \leq i \leq d, 0 \leq j \leq k-1$, is equal to the number of partitions of $n$ into parts which are not of the form $k t$ with

$t \in[(B \cup(4 d+2) B)-(2 B \cup(2 d+1) B)] \cup[(B \cap(4 d+2) B)-(2 B \cap(2 d+1) B)]$.

Letting $k=1$ and $B=\left\{(2 i-1) 2^{u}(2 d+1)^{v} \mid u, v \geq 0,1 \leq i \leq d\right\}$ in Theorem 2, we find that the set $A=\{m \mid m \neq 2 i-1$ with $1 \leq i \leq d\}$ is not only admissible relative to $B$, but the corresponding set is $A$ itself.

Corollary 2. Let $d \in N$ and $B=\left\{(2 i-1) 2^{u}(2 d+1)^{v} \mid u, v \geq 0,1 \leq i \leq d\right\}$. Then the number of partitions of $n$, where no part which is an element of $B$ appears exactly $2 i-1$ times with $1 \leq i \leq d$, is equal to the number of partitions of $n$ into parts which are not of the form $2 i-1$ with $1 \leq i \leq d$.

Theorem 3. Let $k \in N, B \subseteq N$ such that $2 B \subseteq B$. Then the number of partitions of $n$, where no part which is an element of $B$ appears exactly $(2 i-1) k+j$ times with $1 \leq i, 0 \leq j \leq k-1$, is equal to the number of partitions of $n$ into parts which are not of the form $k t$ with $t \in B-2 B$.

Letting $k=1$ and $B=\left\{2^{u}\right\}_{u=v}^{\infty}$ in Theorem 3, we get the following result.

Corollary 3. Let $v \geq 0$ and $B=\left\{2^{u}\right\}_{u=v}^{\infty}$. Then the number of partitions of $n$, where no part which is an element of $B$ appears an odd number of times, is equal to the number of partitions of $n$.into parts which are not equal to $2^{\nu}$.

When $v=0$ in Corollary 3 and when $d=1$ in Corollary 2, the corresponding set is $S=\{i\}_{i=2}^{\infty}$ Thus, we combine these two results to get the following corollary. 
Corollary 4. The number of partitions of $n$, where each power of 2 appears an even number of times, is equal to the number of partitions of $n$ where each part which is a power of 2 times a power of 3 appears at least twice.

In conclusion, we note that, for $m \in N$, the set $B=\{i m\}_{i=1}^{\infty}$ and the set $B$ given in Corollary 2 satisfy the requirements of the three theorems of this paper.

\section{Proofs.}

Proof of Theorem 1. The generating function for $A^{\prime}(n, B)$ is

$$
\begin{aligned}
\left(\prod_{n \notin B}\right. & \left.\frac{1}{1-x^{n}}\right) \prod_{n \in B}\left[\frac{1}{1-x^{n}}-\sum_{i=1}^{d-1} \sum_{j=0}^{k-1} x^{((2 i-1) k+j) n}-\sum_{i=(2 d-1) k}^{\infty} x^{i n}\right] \\
& =\left(\prod_{n \in B} \frac{1}{1-x^{n}}\right) \prod_{n \in B}\left[\frac{1}{1-x^{n}}-\left(\sum_{i=1}^{d-1} x^{(2 i-1) k n}\right)\left(\sum_{j=0}^{k-1} x^{j n}\right)-\sum_{i=(2 d-1) k}^{\infty} x^{i n}\right] \\
& =\left(\prod_{n \in B} \frac{1}{1-x^{n}}\right) \prod_{n \in B}\left[\frac{1}{1-x^{n}}-x^{k n}\left(\frac{1-x^{(d-1) 2 k n}}{1-x^{2 k n}}\right)\left(\frac{1-x^{k n}}{1-x^{n}}\right)-\frac{x^{(2 d-1) k n}}{1-x^{n}}\right] \\
& =\left(\prod_{n \in B} \frac{1}{1-x^{n}}\right) \prod_{n \in B}\left(\frac{1}{1-x^{n}}\right)\left[1-x^{k n}\left(\frac{1-x^{(d-1) 2 k n}}{1+x^{k n}}\right)-x^{(2 d-1) k n}\right] \\
& =\left(\prod_{n \in N} \frac{1}{1-x^{n}}\right) \prod_{n \in B}\left(\frac{1}{1+x^{k n}}\right)\left[\left(1+x^{k n}\right)-x^{k n}\left(1-x^{(d-1) 2 k n}\right)-x^{(2 d-1) k n}\left(1+x^{k n}\right)\right] \\
& =\left(\prod_{n \in N} \frac{1}{1-x^{n}}\right) \prod_{n \in B}\left(\frac{1}{1+x^{k n}}\right)\left(1-x^{2 d k n}\right) \\
& =\left(\prod_{n \in N} \frac{1}{1-x^{n}}\right) \prod_{n \in B} \frac{1-x^{k n}}{1-x^{2 k n}}\left(1-x^{2 d k n}\right) .
\end{aligned}
$$

Now, $2 B \subseteq B \cup 2 d B$. So, all terms of the form $1-x^{2 k n}$, with $n \in B$, cancel with terms of the form $1-x^{k m}$ or $1-x^{2 d k m}$ for some $m \in B$. Also, $B \cap$ $2 d B \subseteq 2 B$. So, if $1-x^{k n}=1-x^{2 d k n^{\prime}}$ with $n, n^{\prime} \in B$, then one of these terms cancels with a term of the form $1-x^{2 k m}$ for some $m \in B$. Hence, this is the generating function for $S(n)$ for some set $S$.

The elements of $N$ which are not in $S$ are those in $(k B \cup 2 d k B)-$ $2 k B$ and those in $k B \cap 2 d k B$. Thus, $S$ consists of all elements of $N$ which are not of the form $k t$ with $t \in[(B \cup 2 d B)-2 B] \cup[B \cap 2 d B]$.

Proof of Corollary 1 . When $d=1$ in Theorem 1 , the restriction on $B$ is that $B \cap 2 B \subseteq 2 B \subseteq B \cup 2 B$. This is clearly satisfied by all $B$. Also, when $d=1$, there is no $i$ such that $1 \leq i \leq d-1$. So, elements of $B$ may appear 
at most $k-1$ times. Additionally, for any $B$,

$$
[(B \cup 2 B)-2 B] \cup[B \cap 2 B]=B .
$$

Proof of Theorem 2. The generating function for $A^{\prime}(n, B)$ is

$$
\begin{aligned}
\left(\prod_{n \xi B}\right. & \left.\frac{1}{1-x^{n}}\right) \prod_{n \in B}\left[\frac{1}{1-x^{n}}-\sum_{i=1}^{d} \sum_{j=0}^{k-1} x^{((2 i-1) k+j) n}\right] \\
& =\left(\prod_{n \in B} \frac{1}{1-x^{n}}\right) \prod_{n \in B}\left[\frac{1}{1-x^{n}}-\left(\sum_{i=1}^{d} x^{(2 i-1) k n}\right)\left(\sum_{j=0}^{k-1} x^{j n}\right)\right] \\
& =\left(\prod_{n \in B} \frac{1}{1-x^{n}}\right) \prod_{n \in B}\left[\frac{1}{1-x^{n}}-x^{k n}\left(\frac{1-x^{2 d k n}}{1-x^{2 k n}}\right)\left(\frac{1-x^{k n}}{1-x^{n}}\right)\right] \\
& =\left(\prod_{n \in B} \frac{1}{1-x^{n}}\right) \prod_{n \in B}\left(\frac{1}{1-x^{n}}\right)\left[1-x^{k n}\left(\frac{1-x^{2 d k n}}{1+x^{k n}}\right)\right] \\
& =\left(\prod_{n \in N} \frac{1}{1-x^{n}}\right) \prod_{n \in B}\left(\frac{1}{1+x^{k n}}\right)\left[\left(1+x^{k n}\right)-x^{k n}\left(1-x^{2 d k n}\right)\right] \\
& =\left(\prod_{n \in N} \frac{1}{1-x^{n}}\right) \prod_{n \in B}\left(\frac{1}{1+x^{k n}}\right)\left(1+x^{(2 d+1) k n}\right) \\
& =\left(\prod_{n \in N} \frac{1}{1-x^{n}}\right) \prod_{n \in B}\left(\frac{1-x^{k n}}{1-x^{2 k n}}\right)\left(\frac{1-x^{(4 d+2) k n}}{1-x^{(2 d+1) k n}}\right) .
\end{aligned}
$$

Now, $2 B \cup(2 d+1) B \subseteq B \cup(4 d+2) B$. So, terms of the forms $1-x^{2 k n}$ and $1-x^{(2 d+1) k n}$, with $n \in B$, cancel with terms of the form $1-x^{k m}$ or $1-x^{(4 d+2) k m}$ for some $m \in B$. In fact, $2 B \cap(2 d+1) B \subseteq B \cap(4 d+2) B$. So, if $1-x^{2 k n}=1-x^{(2 d+1) k n^{\prime}}$ for some $n, n^{\prime} \in B$, then both of these terms cancel with terms $1-x^{k m}=1-x^{(4 d+2) k m^{\prime}}$ for some $m, m^{\prime} \in B$.

$B \cap(4 d+2) B \subseteq 2 B \cup(2 d+1) B$. So, if $1-x^{k n}=1-x^{(4 d+2) k n^{\prime}}$ for some $n, n^{\prime} \in B$, then at least one of these terms cancels with a term of the form $1-x^{2 k m}$ or $1-x^{(2 d+1) k m}$ for some $m \in B$. Hence, this is the generating function for $S(n)$ for some set $S$.

The elements of $N$ which are not in $S$ are those in $[k B \cup(4 d+2) k B]-$ $[2 k B \cup(2 d+1) k B]$ and those in $[k B \cap(4 d+2) k B]-[2 k B \cap(2 d+1) k B]$. Thus, $S$ consists of all elements of $N$. which are not of the form $k t$ with

$$
\begin{aligned}
t \in[(B \cup(4 d+2) B)-(2 B \cup(2 d+1) B)] \\
\cup[(B \cap(4 d+2) B)-(2 B \cap(2 d+1) B)] .
\end{aligned}
$$


Proof of Corollary 2. When

$$
\begin{aligned}
B & =\left\{(2 i-1) 2^{u}(2 d+1)^{v} \mid u, v \geq 0,1 \leq i \leq d\right\}, \\
2 B & =\left\{(2 i-1) 2^{u}(2 d+1)^{v} \mid u \geq 1, v \geq 0,1 \leq i \leq d\right\}, \\
(2 d+1) B & =\left\{(2 i-1) 2^{u}(2 d+1)^{v} \mid u \geq 0, v \geq 1,1 \leq i \leq d\right\}, \quad \text { and } \\
(4 d+2) B & =\left\{(2 i-1) 2^{u}(2 d+1)^{v} \mid u, v \geq 1,1 \leq i \leq d\right\} .
\end{aligned}
$$

So, $2 B \cup(2 d+1) B \subseteq B$ and

$$
B \cap(4 d+2) B=(4 d+2) B=2 B \cap(2 d+1) B .
$$

Thus, $B$ satisfies the requirements of Theorem 2 .

$[B \cap(4 d+2) B]-[2 B \cap(2 d+1) B]$ is the empty set.

$$
\begin{aligned}
{[B} & \cup(4 d+2) B]-[2 B \cup(2 d+1) B]=B-[2 B \cup(2 d+1) B] \\
& =\left\{(2 i-1) 2^{u}(2 d+1)^{v} \mid u, v \geq 0,1 \leq i \leq d \text { and } u, v<1\right\} \\
& =\{(2 i-1) \mid 1 \leq i \leq d\} .
\end{aligned}
$$

Hence, when $k=1, S=N-\{(2 i-1) \mid 1 \leq i \leq d\}=A$.

Proof of Theorem 3. The gen erating function for $A^{\prime}(n, B)$ is

$$
\begin{aligned}
\left(\prod_{n \xi B}\right. & \left.\frac{1}{1-x^{n}}\right) \prod_{n \in B}\left[\frac{1}{1-x^{n}}-\sum_{i=1}^{\infty} \sum_{j=0}^{k-1} x^{((2 i-1) k+j) n}\right] \\
& =\left(\prod_{n \in B} \frac{1}{1-x^{n}}\right) \prod_{n \in B}\left[\frac{1}{1-x^{n}}-\left(\sum_{i=1}^{\infty} x^{(2 i-1) k n}\right)\left(\sum_{j=0}^{k-1} x^{j n}\right)\right] \\
& =\left(\prod_{n \xi B} \frac{1}{1-x^{n}}\right) \prod_{n \in B}\left[\frac{1}{1-x^{n}}-x^{k n}\left(\frac{1}{1-x^{2 k n}}\right)\left(\frac{1-x^{k n}}{1-x^{n}}\right)\right] \\
& =\left(\prod_{n \notin B} \frac{1}{1-x^{n}}\right) \prod_{n \in B}\left(\frac{1}{1-x^{n}}\right)\left[1-\frac{x^{k n}}{1+x^{k n}}\right] \\
& =\left(\prod_{n \in N} \frac{1}{1-x^{n}}\right) \prod_{n \in B}\left(\frac{1}{1+x^{k n}}\right) \\
& =\left(\prod_{n \in N} \frac{1}{1-x^{n}}\right) \prod_{n \in B}\left(\frac{1-x^{k n}}{1-x^{2 k n}}\right) .
\end{aligned}
$$

Now, $2 B \subseteq B$. So, terms of the form $1-x^{2 k n}$ with $n \in B$ cancel with terms of the form $1-x^{k m}$ for some $m \in B$. Hence, this is the generating function for $S(n)$ for some set $S$. 
The elements of $N$ which are not in $S$ are those in $k B-2 k B$. Thus, $S$ consists of all elements of $N$ which are not of the form $k t$ with $t \in B-2 B$.

\section{REF ERENCES}

1. H. L. Alder, Partition identities - from Euler to the present, Amer. Math. Monthly 76 (1969), 733-746. MR 41 \#8366.

2. G. E. Andrews, Number theory, Saunders, Phildelphia, Pa., 1971.

3. J. W. L. Glaisher, Messenger of Mathematics 12(1883), 158-170.

4. E. Moore, Generalized Euler-type partition identities, J. Combinatorial Theory 17 (1974), 78-83.

DEPARTMENT OF MATHEMATICS, CALIFORNIA STATE UNIVERSITY, HUMBOLDT, ARCATA, CALIFORNIA 95521 